\title{
Pre-stack and post-stack seismic for detecting oil and gas based on dual-phase medium theory
}

\author{
Huixing Zhang ${ }^{1, ~ a, ~ P e n g ~ W a n g ~}{ }^{1, b}$, and Yang Sun ${ }^{1, c}$ \\ ${ }^{1}$ Key Lab of Submarine Geosciences and Prospecting Techniques, Ministry of Education, Ocean \\ University of China, Qingdao, China \\ azhhuixing@sina.com, b1193007944@qq.com, ccaesarsy@163.com
}

\begin{abstract}
Keywords: dual-phase medium; pre-stack inversion; hydrocarbon detection; frequency decomposition technology; seismic

Abstract. It is observed that oil and gas reserviors exhibit high seismic wave attenuation. Through extracting the attenuation of seismic wave can detect oil and gas. On the basis of Biot's poroelastic theory, through formula derivation and analysis, we proposed a hydrocarbon detection method based on dual-phase medium theory using pre-stack and post-stack seismic. The inversion results of pre-stack and post-stack field data show that middle offset data for hydrocarbon detection is the best one, and post-stack data take the second place. Near offset data and far offset data are helpful for detecting none and high oil production areas as a supplementary information. Through comparing the results of pre-stack and post-stack data for hydrocarbon detection, we can improve the precision of using seismic to detect oil and gas.
\end{abstract}

\section{Introduction}

Traditional single-phase medium theory simply concerning the existence of solid has played a significant role in structural reservoir detections, while dual-phase medium theory shows an evident superiority in current hydrocarbon detections targeting at lithologic reservoirs, subtle reservoirs and complex reservoirs. Dual-phase medium theory not only assumes that underground media consist of solid and fluid but also involves the relative motion and interaction between solid and fluid. The latest research [1-5] indicates that it is just the relative motion and interaction between solid and fluid that leads to the evident attenuation and dispersion when seismic waves propagating in fluid-bearing media. Therefore, the research on seismic attenuation and dispersion is of great importance to fluid detection $[1,2]$. The dual-phase medium models which describe the relative motion between solid and fluid can be mainly classified into three: Biot model [6], squirt flow model [7] and BISQ model [8]. Biot model properly describes the macro flow between fluid and solid in spite that its attenuation estimation is lower than measured data; squirt flow model places particular emphasis on fluid local motion, thus is sensitive to both the fluid proportion in the porous medium and the shape of the pores. Therefore it requires much more information of the rock structure when applied. However, the details of the rock structure are usually not easy to be obtained, which restrict the application of this theory. BISQ model comprehensively considers both the macro and micro flows between fluid and solid. Nevertheless Bordakov [9] compared Biot theory and BISQ theory and concluded that BISQ theory could not properly describe the relative amplitude feature of fast and slow $\mathrm{p}$ waves in dual-phase medium while Biot theory can get more reliable results. Comprehensive comparison of these three models indicates that Biot model can properly reveal the relative motion between fluid and solid in dual-phase medium and proves to be the suitable equation to describe the dispersion and attenuation in dual-phase medium at present.

Pre-stack seismic data has more lithologic and fluid information compared with post-stack data, therefore hydrocarbon information extracted from pre-stack seismic data is more accurate. In 1970s, applying "bright spot" technique to find gas made a success. Since then, pre-stack attribute inversions like AVO inversion [10,11], elastic impedance inversion [12,13] has been developed and achieved remarkable economic benefits. However, these technologies also have some limits. For example, elastic impedance inversion could not be applied to thin layers[12]; AVO inversion also has some 
failure cases and its precision and reliability still remain to be improved. Therefore, it is important to develop new pre-stack inversion method especially on the basis of dual-phase medium theory.

On the basis of Biot's poroelastic theory, we derived the seismic attenuation coefficient in dual-phase medium and analyzed its characteristic and established the seismic response to hydrocarbon reservoirs. Furthermore, we extracted hydrocarbon information from pre-stack and post-stack field data to detect oil and gas and achieved a result which is consistent with the well data.

\section{Basic theory of hydrocarbon inversion in dual-phase medium}

The $\mathrm{P}$ wave equation of dual-phase medium [6] is

$$
\left\{\begin{array}{l}
\nabla^{2}[(A+2 N) \theta+Q \varepsilon]=\frac{\partial^{2}}{\partial t^{2}}\left(\rho_{11} \theta+\rho_{12} \varepsilon\right)+b \frac{\partial}{\partial t}(\theta-\varepsilon) \\
\nabla^{2}(Q \theta+R \varepsilon)=\frac{\partial^{2}}{\partial t^{2}}\left(\rho_{12} \theta+\rho_{22} \varepsilon\right)-b \frac{\partial}{\partial t}(\theta-\varepsilon)
\end{array} .\right.
$$

Where $A$ and $N$ is elastic coefficients equivalent to lame constants $\lambda$ and $\mu$ in single-phase isotropic medium; $Q$ is the elastic constant of a coupling between the volume change of the solid and that of the fluid; $R$ is the measure of the pressure required on the fluid to force a certain volume of the fluid into the aggregate while the total volume remains constant; $\rho_{11}$ represents the mass of the solid plus the apparent mass due to the relative motion of the solid in the fluid; $\rho_{22}$ represents the mass of the fluid plus the apparent mass due to the relative motion of the fluid in the solid; $\rho_{12}$ represents a mass coupling parameter between fluid and solid; $\theta$ and $\varepsilon$ are the strain of the solid and the fluid respectively, $\theta=\operatorname{div} \mathbf{u}$ and $\varepsilon=\operatorname{div} \mathbf{U}$, where $\mathbf{u}$ and $\mathbf{U}$ are displacements of solid and fluid respectively; $b$ is the dissipation coefficient ; $t$ is propagation time.

Assume that wave propagation in $\mathrm{x}$ direction is

$$
\left\{\begin{array}{l}
u=u_{0} e^{i\left(k^{\prime} x-\omega t\right)}=u_{0} \cdot e^{-\alpha x} \cdot e^{i k x-i \omega t} \\
U=U_{0} e^{i\left(k^{\prime} x-\omega t\right)}=U_{0} \cdot e^{-\alpha x} \cdot e^{i k x-i \omega t}
\end{array} .\right.
$$

Where $u$ is displacement of solid, $U$ is displacement of fluid, $u_{0}$ and $U_{0}$ are initial values of $u$ and $U$ respectively; $k^{\prime}$ is complex wavenumber, $k^{\prime}=k+i \alpha ; k$ is wavenumber, $\alpha$ is attenuation coefficient ; $\omega$ is angular frequency ; $i$ is imaginary unit; $x$ is wave propagation distance.

Substitution in equation (1) and elimination of the exponential yields the relation

$$
\left\{\begin{array}{l}
P(k+i \alpha)^{2} u_{0}+Q(k+i \alpha)^{2} U_{0}=\rho_{11} \omega^{2} u_{0}+\rho_{12} \omega^{2} U_{0}+i b \omega\left(u_{0}-U_{0}\right) \\
Q(k+i \alpha)^{2} u_{0}+R(k+i \alpha)^{2} U_{0}=\rho_{12} \omega^{2} u_{0}+\rho_{22} \omega^{2} U_{0}-i b \omega\left(u_{0}-U_{0}\right)
\end{array},\right.
$$

where $P=A+2 N$.

After derivation we can obtain

$$
\left\{\begin{array}{l}
k^{2}-\alpha^{2}=\frac{\rho_{11} \rho_{22}-\rho_{12}^{2}}{\rho_{12}+\rho_{22}} \cdot \frac{Q+R}{P R-Q^{2}} \cdot \omega^{2} \\
k \alpha=\frac{(P+R+2 Q) \cdot b \cdot \omega}{2\left(P R-Q^{2}\right)}
\end{array} .\right.
$$

Solve equation (4), we get 


$$
\left\{\begin{array}{l}
k=\sqrt{\frac{\sqrt{\left(\rho_{11} \rho_{22}-\rho_{12}^{2}\right)^{2} H^{2} \omega^{4}+\rho^{2} b^{2} H^{2} \omega^{2}}+\left(\rho_{11} \rho_{22}-\rho_{12}^{2}\right) H \omega^{2}}{2 \rho\left(P R-Q^{2}\right)}} \\
\alpha=\sqrt{\frac{\sqrt{\left(\rho_{11} \rho_{22}-\rho_{12}^{2}\right)^{2} H^{2} \omega^{4}+\rho^{2} b^{2} H^{2} \omega^{2}}-\left(\rho_{11} \rho_{22}-\rho_{12}^{2}\right) H \omega^{2}}{2 \rho\left(P R-Q^{2}\right)}}
\end{array} .\right.
$$

Where $H=P+R+2 Q, \rho=\rho_{11}+\rho_{22}+2 \rho_{12}$.

Formula (5) indicates that seismic attenuation coefficient $\alpha$ has relations with medium elastic parameters $(A, N, Q, R)$, density parameters $\left(\rho_{11}, \rho_{22}, \rho_{12}\right)$, dissipation coefficient $b\left(b=\frac{\eta \phi^{2}}{\kappa}\right.$, where $\kappa$ is Darcy's coefficient of permeability, $\eta$ is fluid viscosity, $\phi$ is porosity) and seismic frequency. When underground rock is given, seismic attenuation coefficient is approximately proportional to frequency. That is, when seismic wave propagates in fluid-bearing areas, the relative motion between solid particles and fluid particles leads to seismic attenuation dependent on frequency. Attenuation of lower frequency component of seismic energy is weak while higher frequency component of that is strong which leads to the main frequency of seismic shifts to lower part in dual-phase medium area. Related research [14] indicates that seismic attenuation is not obvious in water-bearing porous rock, while in single-phase medium there is no attenuation. Therefore, the seismic response in oil and gas areas is different from that of none-oil areas. Consequently, we can detect oil and gas by extracting such kind of seismic response from pre-stack and post-stack field data.

\section{Method of hydrocarbon inversion based on dual-phase medium theory}

When seismic wave propagates through hydrocarbon areas, its energy shifts to lower frequency part, which means seismic low frequency energy is relatively enhanced and high frequency energy is weakened in hydrocarbon areas compared with the none hydrocarbon areas. Consequently, it is feasible to extract seismic low frequency energy or high frequency energy to predict the hydrocarbon existence according to whether there is low frequency energy relative enhancement or high frequency energy relative weakness.

Seismic wave low frequency and high frequency energy can be extracted by frequency decomposition technology. In this paper, we use zero-phase triangle recursive filter to implement frequency decomposition on seismic wave energy.

Single trace is of low signal-to-noise ratio, which often leads to larger errors when extracting hydrocarbon information. In order to avoid this problem and reserve incident angle information we partly stack the pre-stack data according to certain offset range. Seismic data can be categorized into three types on the basis of offset range: near offset (small incident angle, within $8^{\circ}$ to $10^{\circ}$ ) data, middle offset (middle incident angle, from $15^{\circ}$ to $25^{\circ}$ ), large offset (large incident angle, from $35^{\circ}$ to $45^{\circ}$ ). Implement stack on these three kinds of offset data respectively and achieve the near offset (small incident angle) data, middle offset (middle incident angle) data and large offset (large incident angle) data. Through frequency decomposition technique, we can get the oil and gas response extracted from pre-stack data. Comprehensively using the oil and gas response from pre-stack (near, middle, far offset) and post-stack seismic data is helpful to improve the precision of hydrocarbon detection.

\section{Hydrocarbon prediction of field data}

Choose the pre and post stack seismic data of one work area. There are nine wells in the work area, with oil well marked with red dots, dry and water wells marked with yellow dots and the unknown wells marked with black dots.

The depth of target layer is from $1300 \mathrm{~m}$ to $1500 \mathrm{~m}$, therefore offset from 0 to $500 \mathrm{~m}$ approximately equals to incident angle within $10^{\circ}$, i.e. small incident angle; offset from $750 \mathrm{~m}$ to $1300 \mathrm{~m}$ approximately equals to incident angle from $10^{\circ}$ to $15^{\circ}$, i.e. middle incident angle; while offset from $1960 \mathrm{~m}$ to $2800 \mathrm{~m}$ 
approximately equals to incident angle from $35^{\circ}$ to $45^{\circ}$, i.e. large incident angle. Consequently choose these three offset ranges to detect oil and gas. The low frequency energy which represents hydrocarbon response of the selected pre-stack data are shown in fig.1-fig.3. Fig.4 presents the hydrocarbon response of post-stack data.

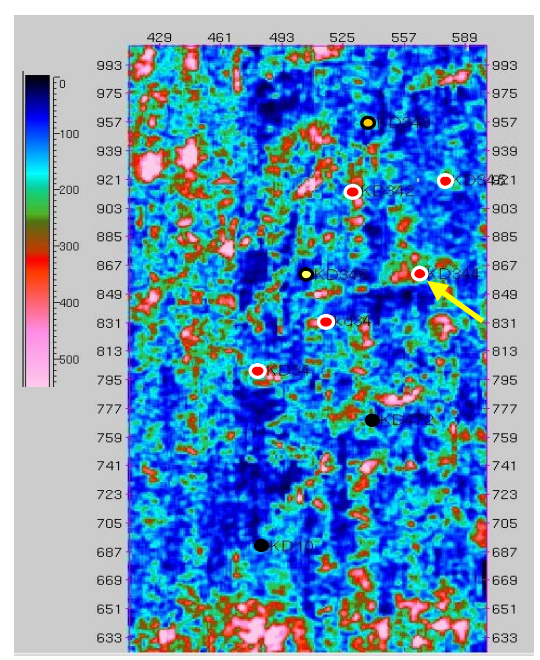

Fig.1 Low frequency energy of near offset: $0-500 \mathrm{~m}$.

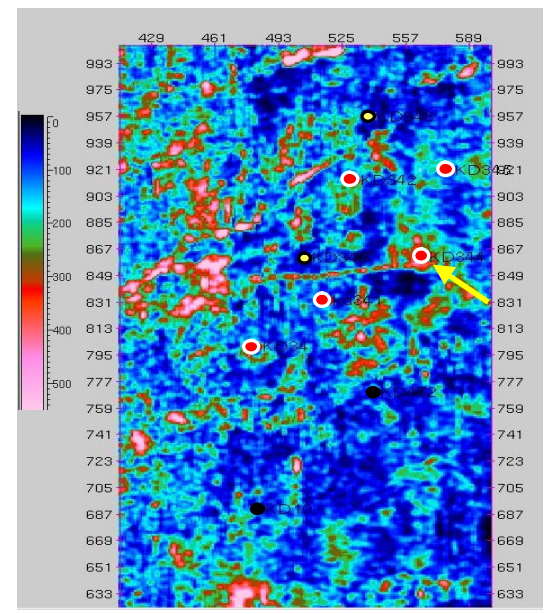

Fig.3 Low frequency energy of far offset: 1960-2800m.

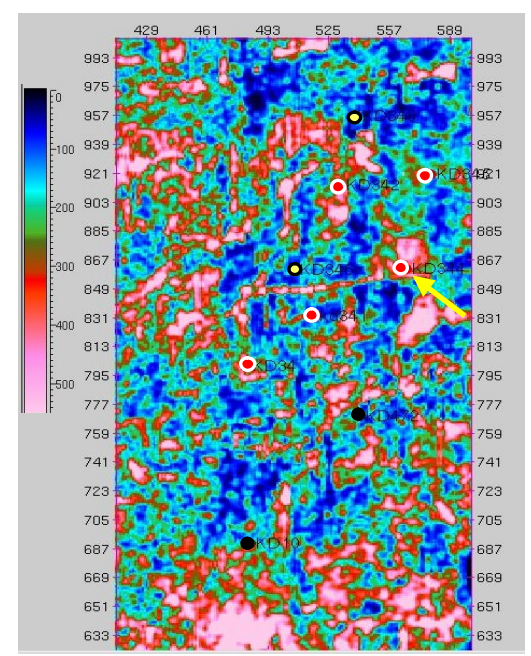

Fig. 2 Low frequency energy of middle offset: $750-1300 \mathrm{~m}$

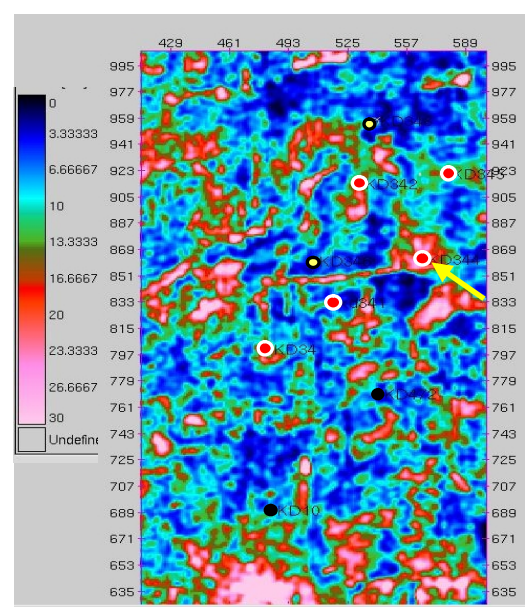

Fig.4 Low frequency energy of post-stack data

Results analysis: Through comparison of fig. 1, fig. 2 and fig. 3, we find that the five oil wells in fig. 3 are all located in red areas (relatively large values) which show that the possibility of containing oil and gas is large. While the two dry wells and water wells are located in blue areas (relatively small values) which shows the small possibility of containing oil and gas. Therefore low frequency energy of middle offset accords with the actual fact best. Fig. 1, fig. 2 and fig. 3 also show that the results of two dry wells and water wells all lie in blue areas in three offset calculations. In addition, the well which an arrow points to as well as its surrounding areas are all red. As we know it is a high productive oil well with oil layer thickness of $7.4 \mathrm{~m}$. Consequently we can conclude as follows: (1) Hydrocarbon response of pre-stack middle offset data coincides with well data best; (2) The area definitely not contains oil and gas if the calculation results in near, middle and far offsets all show none oil; (3) The area is high oil productive if the three offset calculation results all show obvious hydrocarbon existence.

Fig. 3 presents the post-stack hydrocarbon response. Most of the oil wells lie in red areas, while dry wells and water wells all locate in blue areas. Therefore, the hydrocarbon response is basically in accord with well data. Pre-stack seismic data is more sensitive to hydrocarbon existence. So pre-stack middle offset result is more reliable and post-stack data result is helpful as supplementary information. 


\section{Conclusions}

We can detect oil and gas on the basis of dual-phase medium theory through extracting the attenuation of seismic wave. In this paper, we use zero-phase triangle recursive filter to extract seismic attenuation using pre-stack and post-stack seismic data. The inversion results of pre-stack and post-stack field data show that: middle offset data for hydrocarbon detection is the best one, and post-stack data take the second place. Near offset data and far offset data are helpful for detecting none and high oil production area as a supplementary information. Through comparing the results of pre-stack and post-stack data for hydrocarbon detection, we can detect oil and gas in seismic data with high precision and reliability.

\section{Acknowledgements}

This work was financially supported by the National Natural Science Foundation of China (Nos. 41204089 and 41174087).

\section{References}

[1] Müller T M, Gurevich B and Lebedev M. Seismic wave attenuation and dispersion resulting from wave-induced flow in porous rocks - A review J.Geophys. Rev. Vol.75(2010), p.147-164

[2] Zhang H X, and He B S. Propagation and attenuation of P-waves in patchy saturated porous media. Applied Geophysics, Vol.12(2015), p.401-408

[3] Quintal B,H Steeb,M. Frehnerand and S M Schmalholz. Quasistatic finite element modeling of seismic attenuation and dispersion due to wave-induced fluid flow in poroelastic media. $\mathrm{J}$. Geophys. Res. Vol.116(2011), p.B01201

[4] Qi Q M, Müller T M, Gurevich B, et al. Quantifying the effect of capillarity on attenuation and dispersion in patchy-saturated rocks. Geophysics,Vol.79(2014), p.WB35-WB50

[5] Yan F Y, Han D H, Yao Q L, and Zhao L X. Prediction of seismic wave dispersion and attenuation from ultrasonic velocity measurements. Geophysics,Vol.79(2014), p.WB1-WB8

[6] Biot M A. Theory of propagation of elastic waves in a fluid-saturated porous solid. I. Low-frequency range J.Acoust.Soc.Am. Vol. 28(1956), p.168-178

[7] Mavko G and Nur A. Wave attenuation in partially saturated rocks. Geophysics, Vol.44(1979), p. $161-178$

[8] Dvorkin J, Nur A. Dynamic poroelasticity: A unified model with the squirt and the Biot mechanisms, Geophysics,58(1993), p. 524-533

[9] Dvorkin J, Nolen-Hoeksema R and Nur A. The squirt-flow mechanism: Macroscopic description. Geophysics, 59(1994) 428-438

[10] Chiburis E F. Analysis of amplitude versus offset to detect gas/oil contacts in the Arabian Gulf. The 54th annual SEG meeting (Atlanta) Extended abstract, 1984, p.669

[11] Herbert W and Swan. Amplitude versus offset analysis in finely layered media. 58th Annual Int. SEG Meeting , 1988, p.1195-1198

[12] Hu W G, Li F G and Yang H F. Application of Prediction of Pre-stack Elastic Impedance Inversion in Reef-shoal Reservoir at FL Area, Sichuan Basin Mar.Origin Pet. Geol. Vol.15(2010), p. $62-67$

[13] Xiao S H, Li S G and Xu D etc. Application of pre-stack seismic elastic impedance inversion to gas reservoir. Comput.Tech.Geophys.Geochem.Explor, Vol.32(1988) , p.476-479

[14] Li Zishun. Physical mechanism of seismic attenuation in a two-phase medium Appl. Geophysics, Vol. 5(2008), p. 9-17 